\title{
HIGHER DIMENSIONAL GENERALIZATIONS OF THE BLOCH CONSTANT AND THEIR LOWER BOUNDS
}

\author{
BY \\ KYONG T. HAHN(1)
}

\begin{abstract}
A higher dimensional generalization of the classical Bloch theorem depends in an essential way on the "boundedness" of the family of holomorphic mappings considered. In this paper the author considers two types of such "bounded" families and obtains explicit lower bounds of the generalized Bloch constants of these families on the hyperball in the space $\mathbf{C}^{n}$ in terms of universal constants which characterize the families.
\end{abstract}

1. Introduction. The classical Bloch theorem states that if $\&$ is the family of holomorphic mappings $f$ from the unit disc into the complex plane $\mathbf{C}$ such that $\left|f^{\prime}(0)\right|=1$, and if $b(f)$ denotes the least upper bound of the radii of all univalent discs that $f$ carries, then the Bloch constant

$$
\beta(\not)=\inf \{b(f): f \in \mathcal{A}\}
$$

is positive. In this paper we study higher dimensional generalizations of this theorem and obtain explicit lower bounds for the generalized Bloch constants for various families of holomorphic mappings on the hyperball in terms of some universal constants which characterize the given family. The generalized Koebe constants are among such constants. In any effort to generalize the classical theorem of Bloch (or Koebe) to higher dimensional spaces one must first decide a suitable family of holomorphic (or biholomorphic) mappings to work with. Unlike in the case of one complex variable, the usual normalization $(f(0)$ $\left.=0,\left|J_{f}(0)\right|=1\right)$ alone is not enough to guarantee the existence of a positive Bloch (or Koebe) constant. More precisely, the mappings: $w=f_{n}(z)=\left(n z_{1}, z_{2} / n\right)$ map the unit hyperball in $\mathbf{C}^{2}$ onto the circular domains of the form:

$$
n^{-2}\left|w_{1}\right|^{2}+n^{2}\left|w_{2}\right|^{2}<1
$$

which contains no hyperball of positive radius, see $\$ 2$ for more details. In other words, the corresponding family $\&$ in the higher dimensional space $\mathbf{C}^{n}, n>1$, is too vast to expect $\beta(\alpha)>0$.

Received by the editors January 18, 1972 and, in revised form, May 22, 1972.

AMS (MOS) subject classifications (1970). Primary 32A30, 32H99; Secondary 30A32.

Key words and phrases. Classical Bloch theorem, generalized Bloch constant, generalized Koebe constant, holomorphic mapping, the smallest and the largest characteristic values, univalent mapping, quasiconformal holomorphic mapping of order $K$, hyperball, lower bounds of the Bloch constants, Schwarz lemma, Koebe function.

(1) The author would like to take this opportunity to thank Professor S. Kobayashi, and also Professor S. S. Chern for their generosity during his stay at Berkeley. 
In this paper we consider two types of families, $\mathcal{H}_{1}$ and $\mathcal{H}_{11}$, see $\$ 2$ for definitions, and obtain explicit lower bounds of the Bloch constants for $\not_{1}$ in $\$ 3$ and for $\mathcal{H}_{1 I}$ in $\S 4$.

2. Generalized notions of Koebe and Bloch constants. Let $D$ be a bounded homogeneous domain in the space $\mathbf{C}^{n}$ which is star-shaped with respect to $0 \in D . D$ is star-shaped with respect to 0 if $z \in D$ implies $r z \in D$ for $0 \leq r \leq 1$. We denote $D_{r}=\{r z: z \in D\}$ and $\partial D_{r}=$ the boundary of $D_{r}$. In particular, if $D$ is the unit hyperball $B=\{z:|z|<1\},|z|^{2}=\left|z_{1}\right|^{2}+\ldots+\left|z_{n}\right|^{2}$, then $B_{r}$ is just the hyperball of radius $r>0$.

Let $w=f(z)$ be a holomorphic mapping of $D$ into the space $\mathbf{C}^{n}$. The mapping $f$ is univalent in $D$ if it is one-to-one in $D$. To each point $z^{0} \in D$ we associate the number $d_{f}\left(z^{0}\right)$ as follows:

$d_{f}\left(z^{0}\right)=\sup \{r>0:$ there exists a subdomain $G \subset D$ such that

$$
\left.f \text { is univalent in } G \text { and } f\left(z^{0}\right)+D_{r} \subset f(G)\right\} \text {, }
$$

if $J_{f}\left(z^{0}\right) \neq 0$, and $d_{f}\left(z^{0}\right)=0$, otherwise; here $J_{f}\left(z^{0}\right)$ denotes the Jacobian of $f$ at $z^{0}$ and $f\left(z^{0}\right)+D_{r}=\left\{f\left(z^{0}\right)+r \zeta: \zeta \in D\right\}$. Then $d_{f}(z)$ is a nonnegative continuous function of $z \in D$.

For each family \& of holomorphic mappings $f: D \rightarrow \mathbf{C}^{n}$, we formally define the (generalized) Bloch constant of $D$ relative to $\mathcal{H}$, or in brief, the Bloch constant of $\mathcal{H}(D)$, by

$$
\beta(\mathcal{H}) \equiv \beta(\mathcal{H}, D)=\inf \{b(f): f \in \mathcal{H}\}
$$

where

$$
b(f)=\sup \left\{d_{f}(z): z \in D\right\} .
$$

For each $t \in D$ we also define

$$
\beta(\mathcal{H}, t)=\inf \left\{d_{f}(t): f \in \mathcal{H}\right\} .
$$

Then

$$
\beta(\alpha, t) \leq \beta(\alpha) \text { for all } t \in D \text {. }
$$

If $\mathcal{H}_{1}$ and $\mathcal{H}_{2}$ are two families of holomorphic mappings $f: D \rightarrow \mathbf{C}^{n}$ with $\mathscr{H}_{1} \subset \mathcal{H}_{2}$, then

$$
\beta\left(\not_{2}\right) \leq \beta\left(\mathscr{t}_{1}\right)
$$

Let $\delta$ be the family of univalent holomorphic mappings $f: D \rightarrow \mathbf{C}^{n}$ with the following normalization conditions at 0 : 


$$
\begin{aligned}
f(0) & =0, \\
\left|J_{f}(0)\right| & =1 .
\end{aligned}
$$

The (generalized) Koebe constant of $D$ relative to $\delta$, or the Koebe constant of $S(D)$, is defined to be the following number:

$$
\kappa(\delta) \equiv \kappa(\delta, D)=\sup \left\{r>0: D_{r} \subset \bigcap_{f \in \delta} f(D)\right\}
$$

We note that

$$
\kappa(\delta)=\beta(\delta, 0) \leq \beta(\delta) .
$$

A standard normal family argument shows that $\kappa(\delta)$, and hence $\beta(\delta)$ are positive if the family $\delta$ is bounded, see [2, Lemma 3]. On the other hand, a simple example shows that if $\delta$ is unbounded, then $\kappa(\delta)=0$ and, also, $\beta(\alpha)=0$ for any family $\&$ of holomorphic mappings which contains $\delta$. The following example demonstrates this phenomenon:

Let $f_{n}: B \rightarrow C^{2}$, where $B \subset \mathbf{C}^{2}$, be a sequence of biholomorphic mappings given by $f_{n}(z)=\left(n z_{1}, z_{2} / n\right)$ [5]. Clearly, $f_{n}(0)=0$ and $J_{f_{n}}(0)=1$. Furthermore, $\left|w_{1}\right|^{2} / n^{2}+n^{2}\left|w_{2}\right|^{2}<1$ on $B$. Thus, the image domains $f_{n}(B)$ degenerate to the complex plane: $w_{2}=0$ as $n \rightarrow \infty$. Hence, $\bigcap_{n} f_{n}(B)$ contains no hyperball of a positive radius, which clearly implies $\kappa(\delta)=0$. It is also clear that $\beta(\delta)=0$. Hence, by (4), $\beta(\mathcal{A})=0$ if $\delta \subset \mathcal{A}$. From this remark, the Bloch constant $\beta(\mathcal{A})$ being positive depends on the "boundedness" of the family $\&$.

By $\delta(K)$ we denote the bounded family of univalent holomorphic mappings $f: D \rightarrow D_{K}$ satisfying (5a) and (5b). For this family $\delta(K)$ we simply let $\kappa(K)=\kappa(\delta(K))$. It is often convenient to work with the subfamily $\delta_{0}(K)$ $\subset \delta(K)$ with (5b) replaced by

$$
(d f / d z)_{0}=I_{n}
$$

where $(d f / d z)_{0}$ denotes the Jacobian matrix of $f$ at $z=0$, and $I_{n}$ the $n \times n$ identity matrix. For the Koebe constant of $\delta_{0}(K)$, we use $\kappa_{0}(K)=\kappa\left(\delta_{0}(K)\right)$.

We note that $\kappa(\delta, D) \leq 1$, as it follows easily from the generalized KoebeFaber distortion theorem [2, Theorem 6]. (In [2] we used $r_{0}(D)$ for the Koebe constant of $D$ relative to $\delta$.)

Let $\lambda_{f}^{2}$ and $\Lambda_{f}^{2}$ denote the smallest and the largest characteristic values of the hermitian matrix $A^{*} A$, where $A \equiv A_{f}=(d f / d z)$ is the Jacobian matrix of $f$ and $A^{*}$ the conjugate transpose of $A$. In this paper we consider the following two types of families:

$\mathcal{H}_{I} \equiv \mathcal{H}_{1}(K)=$ the family of holomorphic mappings $f: \bar{D} \rightarrow \mathbf{C}^{n}$ such that there exists a constant $K>0$ with 


$$
\max _{z \in \partial D_{r}} \Lambda_{f}(z) \leq K, \quad 0 \leq r \leq 1 .
$$

$\mathscr{H}_{\mathrm{II}} \equiv \mathcal{H}_{\mathrm{II}}(K)=$ the family of holomorphic mappings $f: \bar{D} \rightarrow \mathbf{C}^{n}$ such that there exists a constant $K>0$ with

$$
\max _{z \in \partial D_{r}} \Lambda_{f}(z) \leq K \max _{z \in \partial D_{r}} \lambda_{f}(z), \quad 0 \leq r \leq 1 .
$$

H. Wu ([1], [5]) calls the mapping $w=f(z)$ satisfying (II) for some $K>0$ in the unit hyperball $\bar{B}$ a quasi-conformal holomorphic mapping of order $K$ and shows essentially that every such mapping $f$, subject to the condition $\left|J_{f}(0)\right| \geq a>0$, carries a univalent hyperball of positive radius. The Bloch constant of this family is then the largest among such positive numbers.

Remarks. (1) In the case of one complex variable, each holomorphic mapping $f$ on the unit disc is a $K$-quasi-conformal mapping with $K=1$.

(2) Under the condition (II), $\left|J_{f}(0)\right| \geq a>0$ coincides essentially with the following condition:

$$
\lambda_{f}(0) \geq a>0 \text {. }
$$

Indeed, from $\left|J_{f}(0)\right| \geq \lambda_{f}^{n}(0)$ we know that $\lambda_{f}(0) \geq a$ implies $\left|J_{f}(0)\right| \geq a^{n}>0$. Conversely, $a \leq\left|J_{f}(0)\right| \leq \Lambda_{f}^{n}(0)$ together with $\Lambda_{f}(0) \leq K \lambda_{f}(0)$ implies $\lambda_{f}(0)$ $\geq a^{1 / n} K^{-1}>0$.

3. Lower bounds for the Bloch constants of $\mathscr{H}_{1}$. In this section, we determine lower bounds of the Bloch constants on the hyperball relative to the family $\mathcal{H}_{1}$ satisfying certain normalization conditions at $z=0$.

We begin with the following lemma:

Lemma. Let $w=f(z)$ be a holomorphic mapping defined in a neighborhood of a point $t \in \mathbf{C}^{n}$ into $\mathbf{C}^{n}$ with $J_{f}(t) \neq 0$. Suppose that $\lambda_{f} \equiv \lambda_{f}(t)$ is the positive square root of the smallest characteristic value of the matrix $A^{*} A$ at $t$, where $A \equiv(d f / d z)$. Then the following hold:

(a) The mapping $w=f(z)$ is univalent in any open convex subset $K, t \in K$, of the set

$$
\Omega_{f}=\left\{z:\|A(z)-A(t)\|<\lambda_{f}\right\}
$$

where $\|A\|=\sup _{|x|=1}|A x|$ and $|x|$ denotes the euclidean norm of the n-vector $x$.

(b) If $r_{0}$ is the radius of the largest hyperball contained in $\Omega_{f}$ centered at $t$, then $f\left[B\left(t, r_{0}\right)\right]$ contains the hyperball of radius $r_{0} \lambda_{f} / 2$ centered at $f(t)$, where $B\left(t, r_{0}\right)$ $=\left[z:|z-t|<r_{0}\right]$.

This lemma is a slight improvement over the result obtained by Takahashi [3]. We include here a proof for the sake of completeness.

Proof. Let $z$ and $z^{\prime}$ be any two points in $K$ and let $\sigma$ be the line segment which joins $z$ and $z^{\prime}$ in $K$. Then, by [4, p. 44], 


$$
f(z)-f\left(z^{\prime}\right)=\int_{0}^{1} A\left(z^{\prime}+s\left(z-z^{\prime}\right)\right)\left(z-z^{\prime}\right) d s .
$$

Let $\zeta=z^{\prime}+s\left(z-z^{\prime}\right)$, and rewrite (2) as follows:

$$
f(z)-f\left(z^{\prime}\right)=A(t)\left(z-z^{\prime}\right)+\int_{0}^{1}(A(\zeta)-A(t))\left(z-z^{\prime}\right) d s .
$$

The triangle inequality together with the Schwarz inequality leads to

$$
\left|f(z)-f\left(z^{\prime}\right)\right| \geq\left|A(t)\left(z-z^{\prime}\right)\right|-\int_{0}^{1}\|A(\zeta)-A(t)\|\left|z-z^{\prime}\right| d s .
$$

Since $\sigma$ is a compact subset of $K$, for all $\zeta \in \sigma$,

$$
\|A(\zeta)-A(t)\|<\lambda_{f}(t)(1-\epsilon)
$$

for sufficiently small $\epsilon>0$. Thus,

$$
\begin{aligned}
\left|f(z)-f\left(z^{\prime}\right)\right| & \geq \lambda_{f}(t)\left|z-z^{\prime}\right|-\lambda_{f}(t)(1-\epsilon)\left|z-z^{\prime}\right| \\
& =\epsilon \lambda_{f}(t)\left|z-z^{\prime}\right|>0,
\end{aligned}
$$

from which $f(z)$ is univalent in $K$. Inequality (6) follows from (4) when we use (5) and

$$
\left|A(t)\left(z-z^{\prime}\right)\right| \geq \lambda_{f}(t)\left|z-z^{\prime}\right| .
$$

This proves (a). To prove (b), we let $z^{\prime}=t$ in (4). Then,

$$
|f(z)-f(t)| \geq|A(t)(z-t)|-\int_{0}^{1}\|A(\zeta)-A(t)\||z-t| d s .
$$

We write

$$
F(s)=A(t+s(z-t))-A(t)=A(\zeta)-A(t), \quad 0 \leq s \leq 1 .
$$

Then $F(0)=0, \quad F(1)=A(z)-A(t)$ and $\|F(s)\|=\|A(\zeta)-A(t)\|<\lambda_{f}$ for all $s \in[0,1]$. Hence, by the classical Schwarz lemma,

$$
\|F(s)\|=\|A(\zeta)-A(t)\| \leq \lambda_{f}(t) s .
$$

From (7) and (8), we have

$$
|f(z)-f(t)| \geq \lambda_{f}|z-t|-\lambda_{f}\left[s^{2} / 2\right]_{0}^{1}|z-t|=\lambda_{f}|z-t| / 2 .
$$

Thus, $f\left[B\left(t, r_{0}\right)\right] \supset B\left[f(t), \lambda_{f} r_{0} / 2\right]$.

From this lemma we obtain the following theorem which gives some information about the set $\Omega_{f}$ :

Theorem 1. Let $\delta_{0}$ be the family of univalent mappings $w=f(z)$ on the unit hyperball $B$ satisfing (8) of $\$ 2$. If $f \in \mathcal{S}_{0}$, then the set 


$$
\Omega_{f}=\left\{z:\left\|(d f / d z)_{z}-I_{n}\right\|<1\right\}
$$

cannot cover B completely.

Proof. Suppose that $B \subset \Omega_{f}$. By the previous lemma, (b), $f(B)$ contains the hyperball of radius $1 / 2$. Hence,

$$
\kappa_{0} \equiv \kappa\left(\delta_{0}\right) \geq 1 / 2
$$

But we always have that

$$
\kappa_{0} \leq 1 / 4
$$

Indeed, the mapping

$$
w=f(z)=\left(f_{1}(z), \ldots, f_{n}(z)\right)
$$

with

$$
w_{j}=f_{j}(z)=z_{j} /\left(1-z_{j}\right)^{2}, \quad j=1, \ldots, n,
$$

carries $B$ onto the product domain $S_{1} \times \ldots \times S_{n}$ univalently, where $S_{j}$ is the complex plane minus the slit along the negative real axis from $-1 / 4$ to $-\infty$. (Observe that each $f_{j}(z)$ is the Koebe function.) Moreover, $f \in \delta_{0}$. Hence, $\kappa_{0} \leq 1 / 4$ which contradicts inequality (11). Therefore, $\Omega_{f}$ must leave out points of $B$.

Theorem 2. Let $w=f(z)$ be a holomorphic mapping of $\bar{B}_{R}$ in $\mathbf{C}^{n}$ into $\mathbf{C}^{n}$ such that

$$
\Lambda_{f}(r) \equiv \max _{|z|=r} \Lambda_{f}(z) \leq K \text { for all } r, 0 \leq r \leq R
$$

If $\lambda_{f}(0) \neq 0$, then the following hold:

(a) $w=f(z)$ is univalent in the hyperball $B\left(0, r_{0}\right)$ with $r_{0}=R \lambda_{f}(0) / 2 K$.

(b) $f\left[B\left(0, r_{0}\right)\right]$ contains the hyperball $B\left[f(0), r_{0} \lambda_{f}(0) / 2\right]$.

Proof. From (14), we have

$$
\left\|A_{f}(z)-A_{f}(0)\right\| \leq\left\|A_{f}(z)\right\|+\left\|A_{f}(0)\right\|=\Lambda_{f}(z)+\Lambda_{f}(0) \leq 2 K
$$

for all $|z| \leq R$. By the Schwarz lemma, we obtain

$$
\left\|A_{f}(z)-A_{f}(0)\right\| \leq 2 K|z| / R \text { for }|z| \leq R
$$

Indeed, let

$$
g(t)=t^{-1}\left[A_{f}(t z /|z|)-A_{f}(0)\right]
$$


for $t \in \mathrm{C}$ with $0<|t| \leq R$, and $g(0)=0$ for $t=0$. By (15),

$$
\|g(t)\|=\|A(t z /|z|)-A(0)\| /|t| \leq 2 K / R \text { for }|t|=R .
$$

By the maximum principle,

$$
\|g(t)\| \leq 2 K / R \text { for }|t| \leq R .
$$

In particular, let $t=|z| \leq R$. Then,

$$
\|g(|z|)\|=\|A(z)-A(0)\| /|z| \leq 2 K / R,
$$

which implies (16). From (16), we have

$$
\|A(z)-A(0)\|<\lambda_{f}(0)
$$

if $|z|<r_{0}$ with $r_{0}=R \lambda_{f}(0) / 2 K$. By the above lemma, (a), $w=f(z)$ is univalent in $B\left(0, r_{0}\right)$. By the above lemma, (b), $w=f(z)$ maps $B\left(0, r_{0}\right)$ onto a domain which contains $B\left[f(0), r_{0} \lambda_{f}(0) / 2\right]$. This proves the theorem.

Theorem 3. The Bloch constant $\beta_{I}$ of the family $\mathcal{H}_{1}(K)$ of the holomorphic mappings $f: \bar{B}_{R} \rightarrow \mathbf{C}^{n}$, subject to the condition

$$
\lambda_{f}(0) \geq \alpha \text { for some } \alpha>0,
$$

satisfies the following inequalities:

$$
\beta_{\mathrm{I}} \geq R \alpha^{2} / 4 K
$$

and

$$
\beta_{\mathrm{I}} \geq R \alpha^{2} \kappa_{0}(N) / 2 K, \quad N=K / \alpha,
$$

in terms of the Koebe constant $\kappa_{0}(N)$.

Proof. Inequality (19a) is an immediate consequence of Theorem 2. To prove (19b), we define

$$
g(\zeta)=\left[r_{0} A_{f}(0)\right]^{-1}\left[f\left(r_{0} \zeta\right)-f(0)\right] .
$$

Then $g(0)=0,(d g / d \zeta)_{0}=I_{n}$ and $w=g(\zeta)$ is univalent in $B$ by Theorem 2. Thus, $g \in S_{0}(B)$. Moreover, for $\zeta \in B$,

$$
|g(\zeta)| \leq r_{0}^{-1}|| A_{f}^{-1}(0) \|\left|f\left(r_{0} \zeta\right)-f(0)\right| \leq\left|f\left(r_{0} \zeta\right)-f(0)\right| / r_{0} \lambda_{f}(0) .
$$

Applying (2) of $\$ 3$ to the mapping $w=f(z)$ with $z=r_{0} \zeta$ and $z^{\prime}=0$, we have

$$
f\left(r_{0} \zeta\right)-f(0)=\int_{0}^{1} A_{f}\left(s r_{0} \zeta\right) r_{0} \zeta d s, \quad 0 \leq s \leq 1 .
$$


Hence,

$$
\left|f\left(r_{0} \zeta\right)-f(0)\right| \leq r_{0} \int_{0}^{1}\left\|A_{f}\left(s r_{0} \zeta\right)\right\| d s \leq r_{0} K \quad \text { for }|\zeta|<1 .
$$

From (21), we have

$$
|g(\zeta)| \leq r_{0} K / r_{0} \lambda_{f}(0) \leq K / \alpha
$$

Thus, $g(B) \subset B_{N}$ with $N=K / \alpha$. By definition, the Koebe constant satisfies the following inequality:

$$
\kappa_{0} \equiv \kappa_{0}(N) \leq \min _{|\zeta|=1}|g(\zeta)|
$$

or

$$
\kappa_{0} r_{0} \lambda_{f}(0) \leq \min _{|\zeta|=1}\left|f\left(r_{0} \zeta\right)-f(0)\right| .
$$

This implies that the Bloch constant $\beta_{I}$ of the family $\not t_{1}$ must satisfy

$$
\beta_{\mathrm{I}} \geq \kappa_{0} r_{0} \lambda_{f}(0) \geq R \alpha^{2} \kappa_{0}(N) / 2 K
$$

which establishes inequality (19b).

Corollary 1. The Bloch constant of the family $\mathcal{H}_{\mathrm{I}}(K)$ of holomorphic mappings $f: \bar{B} \rightarrow C^{n}$ such that

$$
\left|J_{f}(0)\right|=1
$$

satisfies the following inequalities:

$$
\beta_{\mathrm{I}} \geq 1 / 4 K^{2 n-1}
$$

and

$$
\beta_{\mathrm{I}} \geq \kappa_{0}(N) / 2 K^{2 n-1}, \quad N=K^{n}
$$

Proof. If $f \in \mathcal{H}_{1}(K)$ satisfies (23), then

$$
1=\left|J_{f}(0)\right| \leq \lambda_{f}(0) \Lambda_{f}^{n-1}(0) \leq \lambda_{f}(0) K^{n-1} .
$$

Hence, $1 / K^{n-1} \leq \lambda_{f}(0)$. Inequalities (24a) and (24b) follow from (19a) and (19b), respectively, when we let $R=1$ and $\alpha=K^{1-n}$.

Corollary 2. The Bloch constant of the family $\mathcal{H}_{1}(K)$ of holomorphic mappings $f: \bar{B} \rightarrow \mathbf{C}^{n}$ such that

$$
(d f / d z)_{0}=I_{n}
$$


satisfies the inequalities

$$
\beta_{\mathrm{I}} \geq 1 / 4 K
$$

and

$$
\beta_{\mathrm{I}} \geq \kappa_{0}(N) / 2 K, \quad N=K
$$

\section{Lower bounds for the Bloch constants of th $_{1 I}$.}

Theorem 4. The Bloch constant $\beta_{\mathrm{II}}$ of the family $\mathcal{H}_{\mathrm{II}}(K)$ of $K$-quasi-conformal holomorphic mappings $f: \bar{B}_{R} \rightarrow \mathbf{C}^{n}$, subject to the condition (18) of $\S 3$, satisfies the following inequalities:

$$
\beta_{\mathrm{II}} \geq \alpha R / 4(2 K+1)
$$

and

$$
\beta_{\text {II }} \geq \alpha R \kappa_{0}(N) / 2(2 K+1), \quad N=2 K,
$$

in terms of the Koebe constant $\kappa_{0}(N)$.

Proof. Following the method used in [3], we define

$$
\varphi(r)=\lambda_{f}(r)(R-r)
$$

where

$$
\lambda_{f}(r)=\max _{|z|=r} \lambda_{f}(z), \quad 0 \leq r \leq R .
$$

Then $\varphi(0)=\lambda_{f}(0) R \geq \alpha R$ and $\varphi(R)=0$. Since $\varphi(r)$ is a continuous function on $[0, R]$, there exists $r_{0} \in[0, R]$ such that $\varphi\left(r_{0}\right)=\alpha R$ and $\varphi(r)<\alpha R$ for $r_{0}<r$ $\leq R$. Choose $z^{0},\left|z^{0}\right|<R$ with $\left|z^{0}\right|=r_{0}$, such that

$$
\varphi\left(r_{0}\right)=\lambda_{f}\left(r_{0}\right)\left(R-r_{0}\right)=\alpha R .
$$

Then,

$$
\lambda_{f}\left(r_{0}\right)=\alpha R /\left(R-r_{0}\right) .
$$

Let $\tau=\left(R-r_{0}\right) / 2$. Then $B\left(z^{0}, \tau\right) \subset B\left(0,\left(R+r_{0}\right) / 2\right) \subset B(0, R)$, since if $z$ $\in B\left(z^{0}, \tau\right)$, then $|z| \leq\left|z^{0}\right|+\left|z-z^{0}\right| \leq r_{0}+\tau=\left(R+r_{0}\right) / 2<R$. Set

$$
g(\zeta)=\left[\tau A_{f}\left(z^{0}\right)\right]^{-1}\left[f\left(z^{0}+\tau \zeta\right)-f\left(z^{0}\right)\right], \quad|\zeta| \leq 1,
$$

where $A_{f}(z)=(d f / d z)_{z}$. We note that $A_{f}^{-1}\left(z^{0}\right)$ exists, since $\left|\operatorname{det} A_{f}\left(z^{0}\right)\right| \geq \lambda_{f}^{n}\left(z^{0}\right)$ $>0$. Observe that

$$
(d g / d \zeta)=A_{f}^{-1}\left(z^{0}\right) A_{f}(\xi), \quad \xi=z^{0}+\tau \zeta
$$


we have

$$
\Lambda_{g}(\zeta)=\left\|A_{g}(\zeta)\right\| \leq\left\|A_{f}^{-1}\left(z^{0}\right)\right\|\left\|A_{f}(\xi)\right\| \leq \Lambda_{f}(\xi) / \lambda_{f}\left(z^{0}\right)
$$

Hence,

$$
\Lambda_{g}(\zeta) \leq K \lambda_{f}\left(\left(R+r_{0}\right) / 2\right) \lambda_{f}^{-1}\left(z^{0}\right) \leq 2 K
$$

The first inequality in (8) follows from inequality (II) and the second inequality follows from (4) and (2a) with $\varphi(r)<\alpha R$. From (6), we also have: $\Lambda_{g}(0)=\lambda_{g}(0)$ $=1$. Thus,

$$
\left\|A_{g}(\zeta)-A_{g}(0)\right\| \leq \Lambda_{g}(\zeta)+\Lambda_{g}(0) \leq 2 K+1 \text { for }|\zeta| \leq 1
$$

By the Schwarz lemma, see (16) of $\S 3$,

$$
\left\|A_{g}(\zeta)-A_{g}(0)\right\| \leq(2 K+1)|\zeta| \text { for }|\zeta| \leq 1
$$

or,

$$
\left\|A_{g}(\zeta)-I_{n}\right\|<1 \text { for }|\zeta|<1 /(2 K+1)
$$

By the lemma, $w=g(\zeta)$ maps $B[0,1 /(2 K+1)]$ univalently onto a domain containing the hyperball $B[0,1 /(4 K+2)]$. From (5), $w=f(z)$ maps the subdomain $B\left[z^{0},\left(R-r_{0}\right) /(4 K+2)\right]$ of $B(0, R)$ univalently onto a domain containing the hyperball $B\left[f\left(z^{0}\right), \alpha R / 4(2 K+1)\right]$. This proves (la). To obtain (2b), we let

$$
h(\zeta)=\left[s A_{g}(0)\right]^{-1}[g(s \zeta)-g(0)], \quad s=1 /(2 K+1) .
$$

Then $h(0)=0,(d h / d \zeta)_{0}=I_{n}$ and $w=h(\zeta)$ is univalent in $B$, since $g$ is univalent in $B(0, s)$. Thus, $h \in \delta_{0}(B)$. Moreover,

$$
|h(\zeta)| \leq|g(s \zeta)-g(0)| / s \lambda_{g}(0)=|g(s \zeta)-g(0)| / s,
$$

from (6). Applying (2) of $\S 3$ for the mapping $g$ with $z=s \zeta$ and $z^{\prime}=0$, we have

$$
g(s \zeta)-g(0)=\int_{0}^{1} A_{g}(t s \zeta) s \zeta d t
$$

The Schwarz inequality yields

$$
|g(s \zeta)-g(0)| \leq \int_{0}^{1}\left\|A_{g}(t s \zeta)\right\| s|\zeta| d t \leq s \int_{0}^{1} \Lambda_{g}(t s \zeta) d t
$$

for $|\zeta| \leq 1$. From (8),

$$
|g(s \zeta)-g(0)| \leq 2 K s=2 K /(2 K+1),
$$


and from (13),

$$
|h(\zeta)| \leq 2 K / s(2 K+1)=2 K
$$

By definition, the Koebe constant satisfies the following inequalities:

$$
\begin{aligned}
\kappa_{0} \equiv \kappa_{0}(2 K) & \leq \min _{|\zeta|=1}|h(\zeta)|=(2 K+1) \min _{|\zeta|=1}|g(s \zeta)| \\
& \leq(2 K+1) \min _{|\zeta|=1}\left|f\left(z^{0}+\tau \zeta\right)-f\left(z^{0}\right)\right| / \tau \lambda_{f}\left(z^{0}\right)
\end{aligned}
$$

Hence,

$$
\max _{\left|\xi-z^{0}\right|=\tau}\left|f(\xi)-f\left(z^{0}\right)\right| \geq \tau \lambda_{f}\left(z^{0}\right) \kappa_{0} /(2 K+1)=\alpha R \kappa_{0} / 2(2 K+1),
$$

from which we have (lb).

Corollary 1. The Bloch constant of the family $\mathcal{H}_{\mathrm{II}}(K)$ of $K$-quasi-conformal holomorphic mappings $f: \bar{B} \rightarrow \mathbf{C}^{n}$ such that $\left|J_{f}(0)\right|=1$ satisfies the following inequalities:

$$
\beta_{\mathrm{II}} \geq K^{1 / n} / 4 K(2 K+1) \geq 1 / 4 K(2 K+1)
$$

and

$$
\beta_{\text {II }} \geq K^{1 / n} \kappa_{0}(2 K) / 2 K(2 K+1) \geq \kappa_{0}(2 K) / 2 K(2 K+1)
$$

Proof. By inequality (II) with $z=0$,

$$
1=\left|J_{f}(0)\right| \leq \lambda_{f}(0) \Lambda_{f}^{n-1}(0) \leq K^{n-1} \lambda_{f}^{n}(0),
$$

and, hence,

$$
K^{(1-n) / n} \leq \lambda_{f}(0)
$$

Inequalities (17a) and (17b) follow from (la) and (lb) with $\alpha=K^{(1-n) / n}$ and $R=1$ when we observe $K \geq 1$.

Corollary 2. The Bloch constant of the family of $K$-quasi-conformal holomorphic mappings $f: \bar{B} \rightarrow \mathbf{C}^{n}$ such that $(d f / d z)_{0}=I_{n}$ satisfies the following inequalities:

$$
\beta_{\mathrm{II}} \geq 1 / 4(2 K+1)
$$

and

$$
\beta_{\mathrm{II}} \geq \kappa_{0}(2 K) / 2(2 K+1)
$$

In particular, if $n=1$, then $\beta_{\mathrm{II}}>1 / 12$. 
Finally, from Theorem 4, we have the following corollary:

Corollary 3. For any $f \in \mathcal{H}_{11}(K)$, defined on $\mathbf{C}^{n}$ and normalized by (18) of $\S 3$, there exists a domain $D \subset C^{n}$ in which $w=f(z)$ is univalent and such that $f(D)$ contains the hyperballs of arbitrarily large radii.

\section{REFERENCES}

1. R. E. Greene and H. Wu, Bloch's theorem for meromorphic functions, Math. Z. 116 (1970), 247 257. MR 42 \#4777.

2. K. T. Hahn, Subordination principle and distortion theorems on holomorphic mappings in the space $C^{n}$, Trans. Amer. Math. Soc. 162 (1971), 327-336.

3. S. Takahashi, Univalent mappings in several complex variables, Ann. of Math. (2) 53 (1951), 464-471. MR 12, 818.

4. V. S. Vladimirov, Methods of the theory of functions of several complex variables, "Nauka", Moscow, 1964; English transl., M.I.T. Press, Cambridge, Mass., 1966. MR 30 \# 2163; MR 34 \# 1551.

5. H. Wu, Normal families of holomorphic mappings, Acta Math. 119 (1967), 193-233. MR 37 \#468.

Department of Mathematics, Pennsylvania State University, University Park, PennSYLVANIA 16802 